\title{
Opposite developmental trends for false recognition of basic and superordinate names
}

\author{
Paula Carneiro \\ Universidade Lusófona de Humanidades e Tecnologias, Lisbon, Portugal \\ Pedro Albuquerque \\ Universidade do Minho, Braga, Portugal \\ Angel Fernandez \\ Universidad de Salamanca, Salamanca, Spain
}

\begin{abstract}
This study investigated the development of false memories for basic and superordinate names using the DRM procedure. Two experiments were conducted with younger (3-5 years old) and older (10-12 years old) children. In the first experiment the DRM procedure was used with categorised lists and in the second experiment both types of lists - categorised and associative - were applied. False recognition for basic and superordinate names showed opposite developmental trends, regardless of list type. False recognition increased for critical-basic items and decreased for critical-superordinate items with age. These opposing results are mainly explained by age differences in conceptual knowledge and editing processes.
\end{abstract}

Keywords: False recognition; Development; Basic and superordinate levels.

Research conducted in the last few decades has shown that we can easily study false memories in the laboratory and that it is possible to remember information that was never experienced. The DRM (after Deese, Roediger, and McDermott) paradigm is one of the procedures that has most contributed to this knowledge, showing very consistent and robust results. This paradigm, first created by Deese (1959) and later rediscovered by Roediger and McDermott (1995), involves the presentation of lists of words (e.g., bed, rest, awake, and so forth) that are highly associated with unpresented converging words (e.g., sleep). In general, this procedure induces high levels of false recall and false recognition of the unpresented converging words (critical items), sometimes at a rate that is similar to the recall or recognition of the studied words.

Recently this paradigm has been applied to children, with results showing that adults and older children produce more false memories of the unpresented critical items than younger children (Anastasi \& Rhodes, 2008; Brainerd, Reyna, \& Forrest, 2002; Brainerd, Reyna, Forrest, \& Karibian, 2006; Carneiro, Albuquerque, Fernandez, \& Esteves, 2007; Holliday \& Weekes, 2006; Howe, 2005, 2006a; Howe, Cicchetti, Toth, \& Cerrito, 2004; Lampinen, Leding, Reed,

Address correspondence to: Paula Carneiro, Rua António de Abreu, 27, 1400-016 Lisboa, Portugal. E-mail: mpcarneiro@hotmail.com

The first author was supported by Fundação para a Ciência e a Tecnologia (SFRH/BPD/26729/2006). Angel Fernandez was supported by the Spanish Ministry of Education and Science and Ministry of Science and Innovation (Projects SEJ2005-03210 and PSI2008-05607, respectively) as well as by Junta de Castilla y León (Project SA031A-06). We would like to acknowledge the comments of three anonymous reviewers. 
\& Odegard, 2006; Metzger et al., 2008; but see also Ghetti, Qin, \& Goodman, 2002, for contrasting results). The same pattern of results has been found even when the lists of words were created by using associative norms for children rather than for adults (Anastasi \& Rhodes, 2008; Carneiro et al., 2007; Metzger et al., 2008). This finding was a surprising result since it is very well known that younger children are, in general, more suggestible than older children and adults (Bruck \& Ceci, 1999; Ceci, 1997; Ceci \& Bruck, 1993; Ceci, Bruck, \& Battin, 2000).

Nevertheless, this kind of result can be very well accommodated by the two most prominent theories of false memories. The thematic consistency framework predicts a developmental increase in false memories when tested by this paradigm because it is thought that the ability of young children to connect the meaning of related words is quite limited. For instance, the fuzzytrace theory (Brainerd \& Reyna, 1998; Reyna \& Brainerd, 1995) assumes that memory representations involve two separate traces: a gist trace, which captures the semantic and relational information of the event, and a verbatim trace, which extracts the specific information in its exact surface form. This theory attributes to gist processing the production of false memories and to verbatim processing the function of suppressing or rejecting false positives, with both types of processing becoming more efficient throughout development (Brainerd, Reyna, \& Poole, 2000). Since the ability to connect meaning across multiple targets increases dramatically with age, and at the same time it is difficult to take advantage of parallel improvements in verbatim memory because of so many meaning-sharing studied targets, this theory predicts that the magnitude of the DRM illusion should increase with development. On the other hand, the activation-monitoring theory (Roediger, Balota, \& Watson, 2001; Roediger \& McDermott, 1995) could initially lead to the prediction that children would show more false memories than adults (Roediger, 1996; Roediger \& McDermott, 2000), because children, like older adults, are usually less accurate in source monitoring; i.e., more prone to confuse different sources of the same phenomenon (Foley, Johnson, \& Raye, 1983). However, in order to account for the general pattern of a developmental increase of false memories obtained with the DRM paradigm, some researchers have more recently advanced an alternative explanation, still in line with the activation framework (Gallo, 2006; Howe, 2006a; Howe, Gagnon, \& Thouas, 2008). Essentially, the argument is that this developmental pattern in false memories could also be explained if one assumes the existence of more elaborate associative and conceptual networks with age, which in turn could lead to a higher activation of the converging words. Howe (2006a) has further suggested that this developmental trend in false memories is not only due to the growth in the knowledge base itself, but could also be explained by an increased automaticity in the activation and accessibility of those concepts and associations in the children's knowledge base.

The DRM procedure has also been used to study false memory for categorical information in adults (e.g., Dewhurst, 2001; Seamon, Luo, Schlegel, Greene, \& Goldenberg, 2000, Exp. 2; Smith, Ward, Tindell, Sifonis, \& Wilkenfeld, 2000) and to a lesser extent in children (Brainerd \& Reyna, 2007; Chiang \& Chiu, 2006; Howe, 2006a; Seamon et al., 2000, Exp. 1). In this case the lists are composed of basic exemplars of categories, presented in a decreasing order of frequency, as dictated by category norms (e.g., Battig \& Montague, 1969). Usually the dominant exemplar of the category is omitted from the study list, serving as a critical item for recall or recognition tests. For example, for the category list composed of cat, lion, giraffe, elephant, and so on, the critical item would be $d o g$. Research using categorised lists has demonstrated that this methodology, similar to the original DRM paradigm, is an efficient tool for evoking false memories for the most dominant exemplars of categories.

Some of these studies (e.g., Buchanan, Brown, Cabeza, \& Maitson, 1999; Park, Shobe, \& Kihlstrom, 2005) were concerned with which type of lists - associative or categorised - produce more false memories, discovering that associative lists produced higher levels of false recognition in adults, a finding also supported by Pierce, Gallo, Weiss, and Schacter (2005) and by Smith, Gerkens, Pierce, and Choi (2002). However, the associative advantage in producing false memories has not been replicated in children. Howe (2006a) showed that associative lists did not differ from categorised lists in producing false recall in children of 5, 7, and 11 years of age. His results showed the expected increase in false recall with age, but this happened regardless of the type of list, indicating that the developmental pattern of false memories for categorised lists is similar to the one observed with the standard application of 
the DRM paradigm. The same developmental pattern for categorised verbal lists was also found by Brainerd and Reyna (2007). Only when the exemplars were presented pictorially did developmental trends in false recall disappear, supporting the results of other studies that also used pictures of exemplars (Howe, 2006a; Seamon et al., 2000) or pictures of associates (Ghetti et al., 2002).

The general developmental pattern of false memories obtained with both associative and categorical lists is usually explained relying only on what happens in the encoding phase, regardless of the underlying theory (fuzzy trace or the more recent version of activation monitoring). In the present study we are also concerned about what happens, in terms of age differences, in the retrieval phase of the false memory phenomenon. Fuzzy-trace theory posits that one specific editing operation, recollection rejection, could suppress false memories of events that did not occur but that are gist-consistent with events that did occur. It is assumed that recollection rejection neutralises the familiarity of false-but-gist-consistent events through the retrieval of true verbatim traces, and that this editing mechanism develops with age (Brainerd \& Reyna, 2002; Brainerd, Reyna, Wright, \& Mojardin, 2003). On the other hand the activation-monitoring theory, relying on Johnson's concept of source monitoring (Johnson, Hashtroudi, \& Lindsay, 1993; Johnson \& Raye, 1981), assumes that, in order to reject a false memory, one should correctly distinguish the memory sources of the presented and critical items, and attribute the origin of a false memory to an internal generation process rather than to an actual, external world event. According to previous research (Foley \& Johnson, 1985; Foley et al., 1983), young children are more likely than adults to confuse memories from different sources, and therefore it is expected that younger children would find it harder to edit out or suppress false memories via monitoring processes.

Gallo (2006) suggested that the underdeveloped monitoring processes of younger children could account for the general observed finding that unrelated false memories are more frequent in younger than in older children: Once the representation of an unpresented item is somehow formed or activated, younger children would be less likely to edit out or suppress its false memory. Following this line of reasoning, it could further be predicted that, under some conditions, younger children could also show more false memories for related or thematic lures than older children. This could happen if the editing process is complicated for younger children because of their lack of appropriate rules for editing out. In more concrete terms, we would like to argue that if the critical and the studied words are from different levels of the categorical hierarchy, false memories should be reduced in a population that could easily distinguish between words of the same category but from different hierarchical levels. However, this would be harder for a population that cannot make use of such knowledge. This argument is based on two types of findings.

The first one is concerned with the relationship between the editing process and distinctiveness. The editing process is usually helped by distinctive information, being more efficient when the unpresented information is in some way distinctive from the studied information. Distinctiveness can help to avoid memory errors (Schacter \& Wiseman, 2006). Schacter, Israel, and Racine (1999) introduced the concept of heuristic distinctiveness to show that participants could engage in a mental operation capable of suppressing false memories based on the recollection of distinctive details. These authors argued that false memories arise when participants are not able to either encode or retain distinctive details of individual items. The encoding of distinctive information, facilitated by presenting pictures as study material, reduces false memories regardless of age (Ghetti et al., 2002; Israel \& Schacter, 1997).

The concept of distinctiveness could explain why some characteristics of the critical lures, when contrasted with the studied words, affect false memories. For example, the length of the critical item was found to be a predictor of false recognition when compared to the length of the studied words (Madigan \& Neuse, 2004). Individuals could easily engage in a thought of the type "I remembered that all the words in the list were short, so a long word might not have been presented". Moreover, emotional critical lures lead to a reduction in false recognition when the studied lists are only composed of neutral words (Pesta, Murphy, \& Sanders, 2001). In the case of the present research, the hierarchical distinctiveness would make it easier to reject the distractors from a different category level. Participants could use a criterion of this type: "I remember that all the items were basic exemplars, thus a superordinate name could have not been in the list". 
The second type of evidence that supports our argument is related to age differences in terms of categorical knowledge. The studies about conceptual development indicate that older children and adults already have a hierarchical structure of their categorical knowledge and that they regularly use this knowledge in memory tasks for a subsequent better retrieval of information (Bjorklund \& Marchena, 1984). In contrast, it is thought that preschool children do not have an actual understanding of hierarchies (Nelson, 1996). Lucariello, Kyratzis and Nelson (1992), working on a variety of tasks, showed that the taxonomic knowledge of preschoolers is restricted to slot-filler categories (i.e., based on a shared event) and that vertical taxonomic knowledge emerges later (as this perspective is not totally consensual: see also other authors, such as Waxman \& Namy, 1997).

Young children can easily perform identification tasks in which they categorise objects at both the basic and the superordinate level, but they cannot succeed in class inclusion tasks at this age. To account for the disparate results obtained in the study of categorical hierarchies with different tasks, another perspective (Blewitt, 1993, 1994) proposed that the understanding of hierarchies is constructed gradually, moving from an implicit to a more explicit knowledge. According to this perspective, preschool children can form superordinate categories, but they cannot draw inferences from hierarchies. Despite different theoretical views, it seems consensual that a hierarchical taxonomic organisation becomes an increasingly dominant mode of classification as children develop (Bjorklund, 2005). Thus for a population that lacks this specific knowledge, as in the case of preschoolers, the suppression of words belonging to a different hierarchical level should be harder or even impossible.

In order to analyse whether children's conceptual knowledge could influence false memory editing, two experiments were designed in which the DRM procedure was applied to categorised lists, aiming to analyse the developmental pattern of false memories for words differing in their levels of categorical hierarchy. False memories will be analysed for words that belong to the same hierarchical level as the studied words (basic) and for words that belong to a different level (superordinate), in two distinct age-groups of children who are presumed to differ in their understanding about the organisation of conceptual knowledge. It is expected that older children will be able to easily edit their false memories for superordinate names, because their knowledge of conceptual hierarchies will increase the distinctiveness of superordinate distractors. In contrast, younger children will be unable to edit those false memories because, besides their usual difficulties in this domain, they lack the conceptual knowledge that would help them in the editing process. Regarding false memories for the basic unpresented exemplars, it is expected that the developmental trend will be the same as the one found for the standard DRM paradigm. This means that older children will be more prone to falsely remember the unpresented basic exemplars than younger children. Both fuzzy-trace theory and the more recent versions of the activation-monitoring framework would predict these results. Both are dual process theories ${ }^{1}$ and integrate error-inflating processes (activation or gist) and error-editing processes (monitoring or recollection rejection) (Arndt \& Gould, 2006). Besides this, both approaches assume that these processes increase with age. Specifically, the improvements in false memory editing are facilitated by developmental progresses either in source monitoring abilities (Foley \& Johnson, 1985; Foley et al., 1983) or in verbatim memory ability (Brainerd \& Reyna, 2002; Brainerd et al., 2003). Thus, both of them would predict that, in general, preadolescents will be more proficient in rejecting false memories than preschoolers. However, in order to provide adequate accounts for the expected results, both theories would need to incorporate the role of distinctiveness afforded by the conceptual knowledge.

\section{EXPERIMENT 1}

Two recent studies, one with adults (Park et al., 2005) and the other with older children (Chiang \& Chiu, 2006), showed that when the critical lure is from another level of categorisation of the list items, false memories are rarely produced. Adults and older children (around 10 years old) usually do not falsely recognise names of categories

\footnotetext{
${ }^{1}$ It is important to note that our own view differs from the associative activation theory (AAT) by Howe $(2005,2006 \mathrm{a})$ in the sense that his theory is not considered a dual-process theory. More in accordance with Gallo (2006), we argue associative activation and also monitoring abilities increase throughout development (this argument will be developed in the General Discussion).
} 
(superordinate level) that are labels of the presented lists of exemplars (basic level) (Chiang \& Chiu, 2006; Park et al., 2005), because those words are from distinct levels of the categorical hierarchy. In our view the distinctiveness of critical words, as a result of their distinct position in the hierarchical level, makes them relatively easy to suppress.

The present experiment addresses this specific question by applying the DRM procedure to categorised lists, testing for both types of critical words - the dominant exemplar (basic level) and the name of the category (superordinate level) in younger (mainly preschoolers) and older children (mainly preadolescents). Specifically, it is predicted that false memories for the dominant exemplar will be higher for older than for younger children, while for the name of the category it is expected that younger children will produce more false memories than older children. The studies by Brainerd, Reyna, and Kneer (1995) and Brainerd and Reyna (1996) support this prediction. Also using categorical material, but a different procedure from the DRM paradigm, these studies showed that 5-year-olds falsely recognised more categorised related words (including category names) than 8-year-olds.

As far as we know, only two other manipulations have demonstrated reverse directions of developmental trends in false memories. Brainerd and Reyna (2007) showed that false memories for a category exemplar increased with age when several exemplars of that category were studied, but decreased when only one exemplar was studied. Dewhurst and Robinson (2004) and Holliday and Weekes (2006) found that false memories increase with age for associative lists but decrease for phonological lists.

\section{Methods}

Participants. A total of 72 children of two agegroups participated in this experiment. The younger group included 36 children from 3 to 5 years of age (17 male, 19 female), with a mean age of 4 years and 3 months; and the older group was composed of 36 children from 10 to 12 years of age ( 23 male, 13 female), with a mean age of 11 years and 8 months. All the participants were Caucasian and native speakers of Portuguese, recruited from kindergartens and elementary schools in Lisbon, Portugal.
Material. Six different categories were selected from the study of Portuguese category norms for children (Carneiro, Albuquerque, \& Fernandez, 2008): Fruits, Animals, Parts of the Human Body, Articles of Clothing, Articles of Furniture, and Vehicles. The 11 most frequent exemplars of each category for preschoolers and for preadolescents were used in this study. The presented lists were formed by the 10 most frequent exemplars (omitting the first exemplar), placed in decreasing order of frequency according to the previously collected norms (Carneiro et al., 2008). The material was specific to each age group - exemplars in the list and their order-and therefore not identical for both ages, since previous studies suggested that children can achieve higher levels in standard memory tasks (Bjorklund \& Thompson, 1983) and in false memory tasks (Carneiro et al., 2007) when the material is specific to their age. Two types of critical words were considered for each list: the name of the category or critical-superordinate (e.g., vehicle) and the first exemplar of each category or criticalbasic (e.g., car). Because the category norms for a Portuguese-speaking population showed that the first exemplar in different ages is not the same for all categories, three of the critical-basic items were different in the two age groups. For the category Fruits the critical-basic item for preschoolers was apple, while it was orange for preadolescents; for the category Parts of the Human Body it was belly for preschoolers and legs for preadolescents; and for the category Animals it was lion for preschoolers and $d o g$ for preadolescents. The remaining three categoriesArticles of Clothing, Articles of Furniture, and Vehicles - had the same critical-basic items - trousers, table, and car - for both ages (see Appendix).

Each participant listened to four out of six lists, with the remaining two lists being used for the recognition task. For each age group, three different groups of participants were formed to ensure that all the lists were counterbalanced between presented and distractor lists.

The recognition test included 24 words: 8 studied words corresponding to the first and fifth positions of each presented list; 4 criticalsuperordinate words of the presented lists; 4 critical-basic words of the presented lists; 4 words from the first and fifth positions of the two unpresented lists; 2 critical-superordinate words of the unpresented lists; and 2 critical-basic words of the unpresented lists. For the critical-superordinate items, the names were simplified to just 
one word in the singular form (fruit, animal, body, clothing, furniture, and vehicle).

Procedure. All the participants were tested individually. Half the participants of each age group performed the recall task after the presentation of each list, whereas the other half did a distractor task after listening to each list. This procedure was adopted because it is known from studies of adults (e.g., Roediger \& McDermott, 1995; Roediger, McDermott, Pisoni, \& Gallo, 2004) as well as from studies of children (Brainerd et al., 2006) that false recognition is enhanced through the performance of a prior recall task of the same material. In both conditions the participants performed a recognition task at the end. In this way it would be possible to analyse whether false recognition is affected by age differences for both the dominant exemplar and the category name, regardless of the recall task.

Participants were randomly assigned to one of the two conditions. In the recall condition the participants were first instructed to listen to the words presented on the tape recorder (at a rate of 2 seconds per word) and were then encouraged to recall the presented words (during $1 \frac{1}{2}$ minutes). In the distractor condition the participants were advised to listen to the same words presented on the tape recorder for a subsequent memory test. Older children were required to count backwards (from 50 to 0 ) after the presentation of each list; and younger children, because of their difficulties in performing this task, were required to count forwards (from 0 to 20). Although the procedure was different, both age groups had the same time to perform it $\left(1 \frac{1 / 2}{2}\right.$ minutes for each list, corresponding to the duration of the recall task). To follow this fixed time it was sometimes necessary to give an additional instruction to either continue or to stop counting. In both conditions the experimenter did not make any reference to the categorical relation between the items. The task was presented as a memory game and the instructions were adapted to the participants' age.

The recognition task was administered after the recall or the distractor task of the last list in a self-paced manner. The participants were instructed to listen to the words read by the experimenter and for each word they answered "yes" if they believed the word had been presented on the tape recorder or "no" if they thought the word had not been presented. To control for order effects, the items of the recognition test were presented in a random order, which was different for each participant. Overall, each experimental session lasted between 15 and 25 minutes.

\section{Results and discussion}

Recall. Correct recall was measured by the proportions of studied words, dividing the total number of correctly recalled words by the number of studied lists and the number of items presented in each list. The false recall was separated in proportions of critical-basic words and in proportions of critical-superordinate words, calculated respectively by dividing the number of critical-basic words and critical-superordinate words falsely recalled by the number of studied lists. The proportion of intrusions was obtained in relation to the number of studied lists and the total number of words recalled for each list. The presented data were collapsed across gender because preliminary analyses showed no significant effects.

Because the false recall of critical-superordinate words was almost non-existent for both age groups (only one young child recalled a superordinate word for one list), the analysis of this type of recall was not taken into consideration in the general analysis of the recall task (see Table 1). The type of recall was then divided into correct recall (studied items), false recall (criticalbasic items), and intrusions. A 2 (Age Group: 3-5 vs $10-12) \times 3$ (Type of Recall: correct vs false vs intrusions) analysis of variance (ANOVA) with repeated measures over type of recall was performed. The analysis revealed two main effects, one of age, $F(1,34)=28.47, M S E=.03, p<.001$, $\eta_{\mathrm{p}}^{2}=.46$, and the other of type of recall, $F(2,68)=$ $56.30, M S E=.03, p<.001, \eta_{\mathrm{p}}^{2}=.62$. Overall, older children recalled more items than younger children. In addition, for the total sample correct recall (.49) was significantly higher than false recall (.26) and intrusions (.08), and false recall was significantly higher than intrusions (all the differences, obtained by pairwise comparisons (Bonferroni test), were significant for $p \leq .001)$. However, the significant Age $\times$ Type of Recall interaction, $F(2$, $68)=20.36, M S E=.03, p<.001, \eta_{\mathrm{p}}^{2}=.37$, clarified that correct and false recall were higher for older children than for younger children, but the reverse pattern occurred in the case of intrusions. Younger children recalled more intrusions than older children (.13 vs .03). This pattern of results with categorical material seems to be in accordance with the developmental pattern observed with 
TABLE 1

Proportions of different types of responses in the recall and recognition tasks of Experiment 1

\begin{tabular}{|c|c|c|c|c|}
\hline & \multicolumn{2}{|c|}{ Younger children } & \multicolumn{2}{|c|}{ Older children } \\
\hline & $M$ & $S D$ & $M$ & $S D$ \\
\hline \multicolumn{5}{|l|}{ Recall } \\
\hline Studied & .30 & .15 & .68 & .10 \\
\hline Critical & .13 & .15 & .39 & .31 \\
\hline Intrusions & .13 & .14 & .03 & .04 \\
\hline \multicolumn{5}{|l|}{ Recognition } \\
\hline \multicolumn{5}{|l|}{ Studied } \\
\hline With recall & $.78(.73)$ & $.18(.16)$ & $.86(.92)$ & $.10(.03)$ \\
\hline Without recall & $.86(.83)$ & $.12(.12)$ & $.71(.77)$ & $.21(.15)$ \\
\hline \multicolumn{5}{|l|}{ Critical-basic } \\
\hline With recall & $.65(.68)$ & $.27(.14)$ & $.64(.78)$ & $.32(.13)$ \\
\hline Without recall & $.61(.66)$ & $.32(.14)$ & $.60(.66)$ & $.30(.15)$ \\
\hline \multicolumn{5}{|c|}{ Critical-superordinate } \\
\hline With recall & $.64(.66)$ & $.29(.16)$ & $.10(.55)$ & $.21(.11)$ \\
\hline Without recall & $.60(.69)$ & $.21(.15)$ & $.46(.62)$ & $.36(.17)$ \\
\hline \multicolumn{5}{|l|}{ Distractors } \\
\hline With recall & .43 & .38 & .01 & .03 \\
\hline Without recall & .31 & .35 & .26 & .25 \\
\hline
\end{tabular}

Data in parentheses are related to A' values

associative material. As had previously been the case for DRM associative lists in other studies (for a review of these studies, see Brainerd, Reyna, \& Ceci, 2008) older children, using categorised lists, also recalled more studied exemplars and false critical-basic items, but made less intrusions than younger children.

In order to distinguish the developmental pattern of intrusions for gist-related and gistunrelated words, the intrusions were divided into related (other exemplars of the category, excluding the dominant exemplar and the category name) and unrelated intrusions (all the other words not considered exemplars of the category, excluding also the dominant exemplar and the category name). The analyses showed that preschoolers gave more unrelated intrusions than preadolescents (respectively $M=.31$ vs $M=.01$ ), $t(34)=2.44, p<.05$, but no significant difference was found for related intrusions $(M=.19$ vs $M=$ $.21), t(34)=.15, p>.05$. The same results were found when the related and unrelated intrusions were divided by the total number of words recalled-for unrelated intrusions: $M=.09$ vs $M=.002, t(34)=2.69, p<.05$; for related intrusions: $M=.05$ vs $M=.03, t(34)=1.42, p>.05$. In line with the findings of other studies (Brainerd et al., 2002; Carneiro et al., 2007), it seems unquestionable that unrelated intrusions decrease with age. For related intrusions we did not find any age differences with categorised lists, although the same type of analyses revealed a decrease of related intrusions for associative lists in other studies (Carneiro et al., 2007). The dissociation between age differences for categorised and associative lists could be related to an increase of knowledge about the number of exemplars that belong to each category. Age differences in related intrusions could be lower when older children know more exemplars of that specific category, which could result in a higher generation of gist-related intrusions. A more elaborate discussion of the related and unrelated intrusions will be included in the general discussion.

Recognition. A preliminary analysis of the recognition of distractors between the different ages revealed that younger and older children differed in terms of their overall response bias, $t(70)=3.28, p<.01$. Younger children recognised more distractor items than older children (.37 vs .14 ), which justifies the use of the signal detection theory for comparisons between those two age groups. Thus, recognition data were corrected by $\mathrm{A}^{\prime}$ values, a signal detection nonparametric measure similar to d' measure that, in this case, gives us information about the ability to discriminate between targets and distractors or criticals and distractors. The method by which signal detection 
measures were computed followed the same procedure used by other authors (see, for example, Odegard, Holliday, Brainerd, \& Reyna, 2008, for a more detailed explanation).

Correct and false recognition were analysed separately. Correct recognition was analysed by a 2 (Age Group: $3-5$ vs $10-12$ ) $\times 2$ (Condition: previous recall vs previous distractor task) between-participants ANOVA. A main effect of age revealed that, in general, older children recognised correctly more items than younger children $\left(.85\right.$ vs $.78 \mathrm{~A}^{\prime}$ values $), F(1,68)=3.96$, $M S E=.02, p<.05, \eta_{\mathrm{p}}^{2}=.06$. However, a significant Age $\times$ Condition interaction, $F(1,68)=$ $17.46, M S E=.02, p<.001, \eta_{\mathrm{p}}^{2}=.20$, showed that older children recognised more correct items when a recall task had been presented previously, whereas younger children had a better performance when the recall task had not been previously administered. This seems to be a striking result since it shows that older children behave more like adults, revealing the same advantage in correct recognition when a recall task is previously administered. Younger children did not benefit from the previous recall and, contrary to what was expected, this task seemed to disrupt the performance in the recognition task. One plausible explanation to account for the different effect of the recall task in distinct age groups could be related to the amount and quality of the items recalled. As younger children usually recall few items and some of these are intrusions, this could increase interference rather than serve as a rehearsal of the studied items. It is possible that the high percentage of intrusions in relation to the items recalled overall could decrease correct recognition in the younger children.

False recognition was analysed by a 2 (Age Group: $3-5$ vs $10-12) \times 2$ (Condition: previous recall vs previous distractor task) $\times 2$ (Critical Item: basic vs superordinate) ANOVA, with repeated measures over type of critical item. This analysis showed a main effect of type of critical item, $F(1,68)=8.01, M S E=.02, p<.01$, $\eta_{\mathrm{p}}^{2}=.11$, with, in general, the critical-basic words producing higher levels of false recognition than critical-superordinate words (.70 vs .63 $\mathrm{A}^{\prime}$ values). In addition, two significant interactions were also found. The significant Age $\times$ Critical Item interaction, $F(1,68)=9.70, M S E=.02, p<.01, \eta_{\mathrm{p}}^{2}=$ .13 , revealed that, compared to younger children, older children produced higher levels of false recognition for the critical-basic words, whereas younger children showed higher levels of false recognition for the critical-superordinate words than did older children. This opposite developmental trend observed for critical-basic and critical-superordinate items was already predicted on theoretical grounds. Although older children produce more false recognition for the criticalbasic items, they are also more prone to suppress the recognition of items that are from a distinct hierarchical level of the studied items. Probably because they are more able to distinguish basic and superordinate names, they could also more easily reject false recognition of unpresented superordinate names. As can be seen in Table 1, older children gave more false recognitions for critical-basic than for critical-superordinate items (.72 vs $.59 \mathrm{~A}^{\prime}$ values), whereas for younger children the means of false recognition for both types of critical items were similar (.67 vs $.68 \mathrm{~A}^{\prime}$ values). This comparison between false recognition of those types of critical items according to the age group seemed to reveal an evolution in conceptual knowledge and editing processes. Because younger children have some limitations in editing and are not helped by the categorical knowledge that makes superordinate names distinctive, they cannot suppress false memories for those items. As a result, they falsely recognised the same amount of critical-basic and criticalsuperordinate items.

The other significant interaction between Critical Item and Condition, $F(1,68)=7.45, M S E=$ $.02, p<.01, \eta_{\mathrm{p}}^{2}=.10$, indicates that, overall, the administration of the recall task before the recognition task enhances false recognition of critical-basic items, but decreases false recognition of critical-superordinate words. The finding that the recall task increases false memory in a subsequent recognition task was already known for adults tested with DRM associative lists (e.g., Roediger \& McDermott, 1995; Roediger et al., 2004). In this experiment the same effect was also found for the dominant unpresented exemplars of categorised lists with a population of children. On the one hand, the recall of list exemplars produced a higher false recognition of the unpresented dominant exemplars of those categories. On the other hand, the recall task stressed the fact that the names of the categories (criticalsuperordinate items) were not presented. This last finding was mainly true for older children, probably because they are more able to distinguish basic from superordinate names. 
In general, the results of Experiment 1 confirmed our prediction of distinct developmental trends for basic and superordinate critical items. Moreover, the application of the DRM procedure to categorised lists showed the same general pattern of age differences as the one already found with the associative lists.

\section{EXPERIMENT 2}

In the first experiment we found that younger children showed more false recognition for the names of the categories than did older children. But is this effect specific to categorised lists, in which the exemplars presented also primed the names of their categories, or is it a more general effect that also happens in associative lists? It seems that, when categorised lists are presented, older children can use their conceptual knowledge to suppress the words that belong to a different level from the studied words. During this task these children probably noticed that the words presented were all exemplars of a basic level of a certain category (e.g., "all words were exemplars of the category animals") and therefore could easily suppress a word (e.g., "animal") that, although related to the category, did not belong to the same hierarchical level. Alternatively, preschoolers might have noticed that all words were related to the same subject, and therefore activated the gist of the list (e.g. "animal") but could not later suppress it because they did not make a distinction between the basic and superordinate levels.

With associative lists participants cannot rely on this type of strategy to suppress the category names, because the lists are not composed of exemplars. We predict that associative lists would decrease the distinctiveness of superordinate names, reducing the probability of those words being rejected. To address this question, categorised and associative lists, sharing the same critical-basic words, were presented to younger and older children to further test the false recognition of those critical-basic words and their corresponding superordinate words. First, this experiment aims to replicate the effect of an opposite developmental trend for basic and superordinate names with categorised lists, and second, it analyses whether this effect could also be extended to associative lists.

This second experiment also makes it possible to compare associative and categorised lists in the production of false memories for basic exemplars in children. The only study that analysed the type of list effect in children's false memories used a recall task (Howe, 2006a), showing similar levels of false recall for associative and categorised lists. The present study also aims to clarify whether it is possible to extend the same results to a recognition task, predicting no differences in false recognition between associative and categorised lists.

\section{Method}

Participants. A total of 56 children participated in this experiment: 28 children from 3 to 5 years of age (11 male, 17 female), with a mean age of 4 years and 8 months, and 28 children from 10 to 12 years of age (11 male, 17 female), with a mean age of 11 years and 9 months. As in Experiment 1, all the participants were Caucasian and native speakers of Portuguese, and were tested in kindergartens and elementary schools in Lisbon. None of these participants took part in Experiment 1 or participated in the normative free association task used to construct the associative material. For the normative study, another 60 participants (30 for each age group) were recruited.

Material. The same six categories selected for Experiment 1 were also used in this experiment as presented lists. The lists were of two types: categorised, and composed of the 10 exemplars of each category, excluding the first element (as in Experiment 1); and associative, and composed of the associates of the dominant exemplars of those categories. Thus, associative and categorised lists shared the same critical-basic items.

In order to construct the Portuguese associative lists for the two age groups a preliminary normative study was conducted. Participants of both ages performed a free association task in which they were asked, for each word, to provide the first three other words that came to their minds. They were instructed to always associate from the target word and not from the first or second associates to minimise response chaining, which could generate associates not directly related with the target word (Nelson, McEvoy, \& Dennis, 2000). The target words included the dominant exemplars of five of the selected categories: apple, lion, belly, trousers, and table for younger children and orange, dog, legs, trousers, and table for older children. The target 
word car was not included because it was already normed in another free association study (Carneiro, Albuquerque, Fernandez, \& Esteves, 2004), and associative lists for both ages were already available for this word. The associates provided for each word were all listed in decreasing order of frequency, and the 10 associates with the highest frequencies were selected to form the associative lists for each age group.

In the memory task each participant listened to six lists: three associative and three categorised. The words from the six presented lists were all different. The lists that served as categorised and associative lists were counterbalanced across participants. Moreover, to control for order effects, half the participants listened first to the associative lists and then to the categorised lists, while the other half listened first to the categorised lists and then to the associative ones.

Two types of recognition tests were created for each age group: one for the associative lists and the other for the categorised lists. Each of these recognition tests was composed of 16 words: 6 words from 3 presented lists (the first and the eighth word of each list), 3 critical-basic words, 3 critical-superordinate words, and 4 unrelated words from one unpresented list.

Procedure. Participants were randomly distributed into four different conditions to control for list and order effects: Group 1 listened first to three associative lists and then to another three categorised lists; Group 2 listened first to the same categorised lists as Group 1 and then to the same associative lists as Group 1; Group 3 listened first to another three associative lists (corresponding to the critical words of the categorised lists of group 1 and 2) and then to other categorised lists (corresponding to the critical words of the associative lists of group 1 and 2); and Group 4 listened first to the same categorised lists as Group 3 and then to the same associative lists as Group 3. All the participants were tested individually.

The participants were initially instructed to listen to the lists of words presented on a tape recorder, at a rate of one word every 2 seconds, for subsequent memory tests. Participants listened to the first three lists (associative or categorised, depending on the group condition), and immediately afterwards they performed the first recognition test relating to those lists (associative or categorised, according to the nature of the presented lists). They subsequently listened to the other three lists, followed by the second corresponding recognition test.

The general instructions for the recognition task were similar to those given in Experiment 1. For each recognition test the participants were also advised to think only about the last three lists presented in order to decide whether the specific word had already been presented or not. As in Experiment 1, the words of the recognition tests were presented in a random order, which was different for each participant. Each experimental session lasted around 15-20 minutes.

\section{Results and discussion}

The mean proportions of studied, critical-basic, critical-superordinate items, and distractors separated by list type and age group are presented in Table 2. Once again, because older children produced fewer false alarms than younger children, $t(70)=2.56, p<.05$, data were corrected by the signal detection theory, providing $\mathrm{A}^{\prime}$ values that are also displayed in parentheses in Table 2.

Correct recognition was analysed by a 2 (Age Group: 3-5 vs 10-12) $\times 2$ (Type of List: associative vs categorised) ANOVA with repeated measures over type of list for the targets $\mathrm{A}^{\prime}$ values. As expected, a main effect of age group, $F(1,54)=$ $11.90, M S E=.006, p<.01, \eta_{\mathrm{p}}^{2}=.18$, revealed that the older group recognised a higher level of correct items than the younger group (.91 vs .86 $\mathrm{A}^{\prime}$ values). There was also a significant Age $\times$ List interaction, $F(1,54)=5.75, M S E=.005, p<.05$, $\eta_{\mathrm{p}}^{2}=.10$, which indicated an opposite advantage of type of list for each age group. The categorised lists lead to an improved correct recognition for the older group, which is consistent with the results usually observed in studies of adults (Buchanan et al., 1999; Pierce et al., 2005). In contrast, the younger group recognised more correct items in the associative lists. Although this last result did not match with the findings of Howe (2006a) regarding a better recall of categorised lists for children of 5, 7, and 11 years of age, it could reflect a preference of younger children for associative relations rather than categorical relations. It is well known that preschool children rely more on associative relations in a wide range of cognitive tasks. For example, picture-sorting tasks have shown that preschool children prefer to organise the figures by associative relations (e.g., bee-honey) than by categorical relations (e.g., bee-butterfly) (e.g., Smiley \& 
TABLE 2

Proportions of different types of responses in the recognition task of Experiment 2

\begin{tabular}{|c|c|c|c|c|}
\hline & \multicolumn{2}{|c|}{ Younger children } & \multicolumn{2}{|c|}{ Older children } \\
\hline & $M$ & $S D$ & $M$ & $S D$ \\
\hline \multicolumn{5}{|l|}{ Studied } \\
\hline Associative & $.80(.88)$ & $.18(.05)$ & $.84(.89)$ & $.17(.07)$ \\
\hline Categorical & $.74(.84)$ & $.21(.12)$ & $.88(.92)$ & $.12(.03)$ \\
\hline \multicolumn{5}{|l|}{ Critical-basic } \\
\hline Associative & $.55(.77)$ & $.31(.12)$ & $.69(.83)$ & $.29(.13)$ \\
\hline Categorical & $.44(.70)$ & $.39(.17)$ & $.67(.84)$ & $.27(.09)$ \\
\hline \multicolumn{5}{|c|}{ Critical-superordinate } \\
\hline Associative & $.43(.70)$ & $.36(.19)$ & $.21(.64)$ & $.24(.15)$ \\
\hline Categorical & $.36(.68)$ & $.29(.15)$ & $.12(.61)$ & $.24(.11)$ \\
\hline \multicolumn{5}{|l|}{ Distractors } \\
\hline Associative & .07 & .12 & .04 & .14 \\
\hline Categorical & .10 & .16 & .01 & .05 \\
\hline
\end{tabular}

Data in parentheses are related to A' values

Brown, 1979). Also, for those children clustering in free recall tasks is mainly dominated by associative criteria, even in situations where categorical criteria would be a better strategy (Bjorklund \& Marchena, 1984).

False recognition was first analysed by a 2 (Age Group: $3-5$ vs. 10-12) $\times 2$ (Type of List: associative vs categorised) $\times 2$ (Critical Item: basic vs superordinate) ANOVA, with repeated measures over type of list and critical item on each participant's A' values. The analysis revealed a main effect of critical item, $F(1,54)=76.28$, $M S E=.012, p<.001, \eta_{\mathrm{p}}^{2}=.59$, with the criticalbasic items producing a higher level of false recognition than the critical-superordinate items (.79 vs .66 $\mathrm{A}^{\prime}$ values). Also, a significant Age $\times$ Critical Item interaction was found, $F(1,54)=$ 29.52, $M S E=.012, p<.001, \eta_{\mathrm{p}}^{2}=.35$, revealing that older children produced more false recognition for the critical-basic items than younger children (.84 vs .74 $\mathrm{A}^{\prime}$ values), whereas younger children falsely recognised more criticalsuperordinate items than older children (.69 vs $.63 \mathrm{~A}^{\prime}$ values). This significant interaction was in line with the results of Experiment 1, and it reinforces the idea that there are different developmental trends for critical-basic and criticalsuperordinate unpresented items, regardless of the type of list. Even for the associative lists, the superordinate names were more likely to be falsely recognised by the younger children than by the older ones. Separated analysis for the critical-basic and for the critical-superordinate items ( 2 age $\times 2$ type of list ANOVA) confirmed that the difference between the younger group and the older one was significant for both types of critical items - for critical-basic items, $F(1,54)=$ $13.64, M S E=.019, p<.01, \eta_{\mathrm{p}}^{2}=.20$; for criticalsuperordinate items, $F(1,54)=5.15, M S E=.023$, $p<.05, \eta_{\mathrm{p}}^{2}=.09$. Moreover, the $2($ age $) \times 2$ (type of list) analysis of variance for the critical-basic items showed a marginal Age $\times$ Type of List interaction, $F(1,54)=3.80, M S E=.014, p=.06$, $\eta_{\mathrm{p}}^{2}=.07$, which suggests that younger children could be more likely to recognise the criticalbasic items for the associative lists than for the categorised lists. Once again, although only a tendency, this result could suggest that, because of their preference for associative relations, it is easier for younger children to extract the associative gist than the categorical-dominant exemplar. For the older group, the false recognition proportions of critical-basic items were similar for both types of list, which is consistent with the false recall results of the study of Howe (2006a). The same type of analysis for the critical-superordinate items did not reveal a significant interaction. No other significant effects emerged.

Overall, this experiment replicated the effect of opposite developmental trends for false recognition of basic and superordinate names with categorical lists, and extended it to associative lists. Besides this, the results showed no significant differences in false recognition for basic names between associative and categorised lists for older children, in line with the false recall results of Howe (2006a). However, preschoolers showed higher true and false recognition for 
associative than for categorised lists, probably reflecting a preference for associative rather than categorical relations. Although those results were not totally in accordance with the results of Howe (2006a), they also contradicted the assumption that the DRM illusion in children is driven by thematic rather than associative information.

\section{GENERAL DISCUSSION}

Both experiments analysed whether names from different levels in the categorical hierarchy produce similar or opposite developmental trends for false memories. These experiments showed that when lists of basic exemplars were presented for study, opposite developmental trends were observed for the unpresented basic dominant exemplar and for the unpresented superordinate category name. There is an increase of false recognition for critical-basic items and a decrease of false recognition for critical-superordinate items with age (an increase of false recall for critical-basic items was also obtained in Experiment 1).

As in the interpretation of the developmental trend for the DRM associative lists, in categorised lists the critical-basic items seem to be more easily activated by older children because of an enriched conceptual knowledge and/or greater ease in connecting the gist. The enhanced strength of links between the exemplars of the categories and the better definition of the dominant exemplar in the category with age could account for the critical-basic developmental trend. But the increasing ability in gist processing by older children could also lead to a better extraction of the mental representation of the category, which in turn could strongly activate its dominant exemplar. As occurred in the DRM associative lists, the explanations of age differences in false memories for DRM categorical lists using the critical-basic items are based on what happens in the encoding phase.

However, the opposite developmental trend observed for critical-superordinate items can be explained by taking into account differences in the editing processes between the two age groups. As far as monitoring abilities are concerned, younger children are in general more likely than adults to confuse memories from different sources. It is well documented that preschool children can use some forms of monitoring, but they are particularly inefficient when the origins of memory are rather similar (Lindsay, Johnson, \& Kwon, 1991), as when they have to discriminate which actor has performed a given action or when they have to distinguish between performed actions and imagined actions (Roberts, 2000). It seems that their lower quality of encoding, and the fact that they do not realise the connection between knowledge and source is important for making accurate attributions, are possible causes of their source confusions (Perner, 2000; Roberts, 2000). Other authors (e.g., Schacter, Kagan, \& Leichtman, 1995) attribute the cause of young children's source-monitoring difficulties to their immature frontal functioning. Another perspective is given by fuzzy-trace theory, an approach that also assumes the involvement of another false-memory editing operation. Brainerd et al. (2003) argued that, although it is available to young children, recollection rejection becomes more efficient with age, and that age-related improvements in the retention of verbatim memories, together with an increase in the effectiveness of retrieval cues, are the factors responsible for the development of this operation.

However, age differences in editing processes are not a sufficient explanation for all the results. Another factor needs to be found to explain why older children show substantial levels of false memories for critical-basic items and very low levels of false memories for critical-superordinate items. It was thought that the distinctiveness given by the understanding of conceptual organisation could provide the central explanation for these results. It is known from earlier studies on conceptual development that preadolescents are already able to discriminate basic from superordinate items (Bjorklund, 2005). The results of the present study suggest that these children can use this information to suppress false recognition for words that belong to a different hierarchical level from the studied words. In this case the distinctiveness provided by the presentation of exemplars, all from the same basic category, facilitated the false memory suppression of other names that, although they are related to the same category, do not belong to the same hierarchical level. In contrast, younger children are not able to do this. Younger children do not distinguish different levels of the hierarchy or at least do not use this knowledge to achieve a more accurate memory performance. Because for these children superordinate names are not made distinctive by conceptual knowledge, they could not 
use a distinctiveness-based heuristic to suppress them.

Although basic names are acquired first in children's language, are more frequent in adult discourse and are used earlier and are more accessible in naming behaviour than superordinate names (Horton \& Markman, 1980; Mervis \& Crisafi, 1982; Skwarchuk \& Anglin, 1997), younger children recognised unpresented superordinate names at the same rate as unpresented basic names. This could mean that, although younger children do not use the hierarchical knowledge in memory tasks, they are able to form mental representations of the categories (including their names) or extract the gist of the lists when basic exemplars are presented. This interpretation is consistent with the Bjorklund (1980) study in which kindergarten children increased performance in a category identification task (prior to recall) when the exemplars were presented in blocks rather than in random order. It seems that, under appropriate presentation conditions, younger children can extract category relations and therefore can activate the name of the categories.

The observation that children can extract category relations and activate the name of the categories does not mean that they could use this information in an editing process. Therefore, it is reasonable to argue that superordinate names are activated at the same rate as the dominant exemplar, but because younger children are limited in editing processes and cannot be helped by the use of hierarchical knowledge, they are not able to suppress false memories for superordinate names. Although this seems to be a coherent explanation, one cannot exclude the possibility that young children do not edit critical-superordinates because they are particularly inefficient in implementing distinctiveness heuristics of all types (Howe, 2006b). Perhaps young children are not able to abstract a general rule as a strategy for rejecting false memories. Actually, it is known that young children's performances decrease when the tasks rely on memory strategies and that only at the end of elementary school years can they conduct appropriate memory searches during retrieval (Bjorklund \& Coyle, 1995; Schneider \& Bjorklund, 1998). Specifically regarding false memories, recent studies (Ghetti, 2003; Ghetti \& Alexander, 2004; Ghetti \& Casteli, 2006) have shown that young children are not able to apply rules based on expected memorability to infer event non-occurrence, even when they are instructed to use them. Thus, the results of the present study do not rule out the alternative argument that young children are able to distinguish different hierarchical levels but are still inefficient in using a decision-based process such as a distinctiveness heuristic.

A similar opposite effect was also observed when the standard DRM procedure with associative lists was used. Because associative lists give rise to a decrease in the distinctiveness of superordinate names, we predicted that the editing process for those lists would be lower than for the categorised lists, resulting in greater false recognition for critical-superordinates of the associative DRM. But we did not find a significant difference in false memories for superordinate names between those two types of lists. For older children this could mean that they are able to discriminate basic from superordinate categories even when distinctiveness decreases; for younger children, this result indicates that they are probably not influenced by such distinctiveness manipulation because they do not have the conceptual knowledge that allows for the emergence of distinctiveness. Even when using associative lists, younger children showed higher levels of false recognition for superordinate names than older children. It seems that, for these children, the confusion between the dominant exemplar and the superordinate name of the same category still occurs in associative lists.

The result that younger children falsely recognise more superordinate names than older children even for associative lists (as well as the similar level of related intrusions obtained in Experiment 1 for both age groups) seems to contradict the assumption that older children are more prone to false memories of all types of semantic gist-related items. In fact, studies that analyse critical and related intrusions together showed an increase of semantic gist-related intrusions with development (Dewhurst \& Robinson, 2004). However, when the analyses exclude the critical items and separate the related from the unrelated intrusions, the same pattern is not always obtained. In the Carneiro et al. (2007) study, preschool children recalled significantly more related and unrelated intrusions than preadolescents (although this is not evident in the study of Brainerd et al., 2002). Furthermore, when the related intrusions were divided by the number of words recalled, preschool children produced more relative false recall compared to adults (Carneiro et al., 2007). This seems to 
reinforce the idea that false memories in older children and adults are much more centred on the critical words (the converging associative word or the dominant exemplar), whereas in younger children they are more dispersed, including all the other related items that the editing processes were not successful in suppressing. Thus, this explanation attributes a special role to the editing processes in the production of false memories in children using the DRM paradigm. While the age trends of false memories for associative converging words or dominant exemplars could be explained only by processes that occur at encoding, without any contribution from the editing processes, the same does not happen when superordinate names are tested. In this case, it is fundamental to refer to inference or decision processes that occur mainly at retrieval. Thus, it seems that both fuzzy-trace and activationmonitoring theories could explain the observed results, since they include an editing process in their frameworks, and they both complement it with distinctiveness-based mechanisms provided by knowledge of the categorical hierarchy.

Actually, relying exclusively on the concept of gist, it would be difficult to explain such results. As was pointed out earlier (Chiang \& Chiu, 2006), fuzzy-trace theory is unspecific about which item - dominant basic or category labelis the best representation of a categorised list. Gist-based explanations are problematic, especially if one accepts that the category name, corresponding to the theme of the list, is considered to be the gist representation. In this case, it would be expected that the category names would produce more false recognition than dominant exemplars, at least for older children. Moreover, because younger children are assumed to be limited in their gist-processing abilities, it would also be expected that older children would recognise more category names than younger children. But contrasting results to these ones were found in the present study.

This study emphasised that not all gist-related items behave in the same way. The developmental direction of false memories for standard critical words (converging associates or dominant exemplars) could be rather distinct from the developmental direction of other gist-related words, such as superordinate names. The findings of the present study suggest that those names could be exceptions, due to the interference of the degree of understanding of hierarchical structures. In fact, although we found a decrease in false memories for category names as age progresses, we did not find significant age differences for the recall of other gist-related words (i.e., related intrusions) with categorised lists. In order to have a definite answer in relation to the developmental trend of false memories for gist-related words, it would be important to know what happens when other types of semantic gist-related words are tested and to compare these with category names and with other words that are considered not to be gist related.

In the present study we used materials based on age-appropriate norms because in this way we can make sure that the exemplars are familiar to children and guarantee the use of dominant exemplars for each age group. This methodology seems to be more appropriate for the study of false memories in children, since earlier experiments showed higher levels of false recall in the case of materials designed specifically for the children's age (Carneiro et al., 2007). However, we are aware that this methodology gives rise to differences in the stimuli used. Similarly, in order to adjust the distractor task to the abilities of young children, these children were instructed to count forwards rather than backwards, as was the case with preadolescents. Although justified, and probably more appropriate, the lack of an equal methodology (for both materials and distractor task) between the different age groups could be considered a limitation of the present study.

In summary, the developmental trend of false memories obtained with the DRM paradigm has been explained by processes that could mainly operate at encoding by both thematic consistency and associative-based activation. These approaches could also explain the same developmental direction for false memories at the basic categorical level. However, in order to account for the false recognition results at the superordinate level, it is crucial to take into account age differences in inferential or decision processes that occur mainly at retrieval. Using categorised lists and a similar DRM procedure, this study emphasised the contribution of the distinctiveness information provided by conceptual knowledge in explaining age differences for false memories of gist-related words.

Manuscript received 26 August 2007 Manuscript accepted 19 January 2009 First published online 25 February 2009 


\section{REFERENCES}

Anastasi, J. S., \& Rhodes, M. G. (2008). Examining differences in the levels of false memories in children and adults using child-normed lists. Developmental Psychology, 44, 889-894.

Arndt, J., \& Gould, C. (2006). An examination of twoprocess theories of false recognition. Memory, 14, 814-833.

Battig, W. F., \& Montague, W. E. (1969). Category norms for verbal items in 56 categories. A replication and extension of the Connecticut category norms. Journal of Experimental Psychology Monograph, 80, 1-46.

Bjorklund, D. (2005). Children's thinking: Cognitive development and individual differences (4th ed.). Belmont, CA: Wadsworth/Thomson Learning.

Bjorklund, D. F. (1980). Children's identification of category relations in lists presented for recall. Journal of Genetic Psychology, 136, 45-53.

Bjorklund, D. F., \& Coyle, T. R. (1995). Utilization deficiencies in the development of memory strategies. In F. E. Weinert \& W. Schneider (Eds.), Memory performance and competencies (pp. 161180). Hillsdale, NJ: Lawrence Erlbaum Associates Inc.

Bjorklund, D. F., \& Marchena, M. R. (1984). Developmental shifts in the basis of organisation in memory: The role of associative versus categorical relatedness in children's free recall. Child Development, 55 , 952-962.

Bjorklund, D. F., \& Thompson, B. E. (1983). Category typicality effects in children's memory performance: Qualitative and quantitative differences in the processing of category information. Journal of Experimental Child Psychology, 35, 329-344.

Blewitt, P. (1993). Taxonomic structure in lexical memory: The nature of developmental change. Annals of Child Development, 9, 103-132.

Blewitt, P. (1994). Understanding categorical hierarchies: The earliest levels of skill. Child Development, 65, 1279-1298.

Brainerd, C. J., \& Reyna, V. F. (1996). Mere memory testing creates false memories in children. Developmental Psychology, 32, 467-478.

Brainerd, C. J., \& Reyna, V. F. (1998). Fuzzy-trace theory and children's false memories. Journal of Experimental Child Psychology, 71, 81-129.

Brainerd, C. J., \& Reyna, V. F. (2002). Recollection rejection: How children edit their false memories. Developmental Psychology, 38, 156-172.

Brainerd, C. J., \& Reyna, V. F. (2007). Explaining developmental reversals in false memory. Psychological Science, 18, 442-448.

Brainerd, C. J., Reyna, V. F., \& Ceci, S.J. (2008). Developmental reversals in false memory: A review of data and theory. Psychological Bulletin, 134, 343-382.

Brainerd, C. J., Reyna, V. F., \& Forrest, T. J. (2002). Are young children susceptible to the false-memory illusion? Child Development, 73, 1363-1377.

Brainerd, C. J., Reyna, V. F., Forrest, T. J., \& Karibian, D. (2006). Development of the false-memory illusion. Developmental Psychology, 42, 962-979.
Brainerd, C. J., Reyna, V. F., \& Kneer, R. (1995). False recognition reversal: When similarity is distinctive. Journal of Memory and Language, 34, 157-185.

Brainerd, C. J., Reyna, V. F., \& Poole, D. A. (2000). Fuzzy-trace theory and false memory: Memory theory in the courtroom. In D. F. Bjorklund (Ed.), False-memory creation in children and adults: Theory, research, and implications (pp. 93-127). Mahwah, NJ: Lawrence Erlbaum Associates Inc.

Brainerd, C. J., Reyna, V. F., Wright, R., \& Mojardin, A. H. (2003). Recollection rejection: False memory editing in children and adults. Psychological Review, 110, 762-784.

Bruck, M., \& Ceci, S. J. (1999). The suggestibility of children's memory. Annual Review of Psychology, 50, 419-439.

Buchanan, L., Brown, N. R., Cabeza, R., \& Maitson, C. (1999). False memories and semantic lexicon arrangement. Brain and Language, 68, 172-177.

Carneiro, P., Albuquerque, P., \& Fernandez, A. (2008). Portuguese category norms for children. Behavior Research Methods, 40, 177-182.

Carneiro, P., Albuquerque, P., Fernandez, A., \& Esteves, F. (2004). Normas de associação livre de 16 palavras portuguesas para crianças de diferentes faixas etárias. Laboratório de Psicologia, 2, 49-76.

Carneiro, P., Albuquerque, P., Fernandez, A., \& Esteves, F. (2007). Analyzing false memories in children with associative lists specific for their age. Child Development, 78, 1171-1185.

Ceci, S. J. (1997). False beliefs: Some developmental and clinical considerations. In D. L. Schacter (Ed.), Memory distortion (pp. 91-125). Cambridge, MA: Harvard University Press.

Ceci, S. J., \& Bruck, M. N. (1993). Suggestibility of the child witness: A historical review and synthesis. Psychological Bulletin, 113, 403-439.

Ceci, S. J., Bruck, M. N., \& Battin, D. B. (2000). The suggestibility of children's testimony. In D. F. Bjorklund (Ed.), False-memory creation in children and adults: Theory, research and implications (pp. 169-201). Mahwah, NJ: Lawrence Erlbaum Associates Inc.

Chiang, W., \& Chiu, C. (2006). Children's category structure and false memories. Psychologia, 49, 178-192.

Deese, J. (1959). On the prediction of occurrence of particular verbal intrusions in immediate recall. Journal of Experimental Psychology, 58, 17-22.

Dewhurst, S. A. (2001). Category repetition and false recognition: Effects of instance frequency and category size. Journal of Memory and Language, 44, 153-167.

Dewhurst, S. A., \& Robinson, C. A. (2004). False memories in children: Evidence for a shift from phonological to semantic associations. Psychological Science, 15, 782-786.

Foley, M. A., \& Johnson, M. K. (1985). Confusions between memories for performed and imagined actions: A developmental comparison. Child Development, 56, 1145-1155.

Foley, M. A., Johnson, M. K., \& Raye, C. L. (1983). Age-related changes in confusion between 
memories for thoughts and memories for speech. Child Development, 54, 51-60.

Gallo, D. A. (2006). Associative illusions of memory: False memory research in DRM and related tasks. New York: Psychology Press.

Ghetti, S. (2003). Memory for nonoccurrences: The role of metacognition. Journal of Memory and Language, 48, 722-739.

Ghetti, S., \& Alexander, K. W. (2004). "If it happened, I would remember it": Strategic use of event memorability in the rejection of false autobiographical events. Child Development, 75, 542-561.

Ghetti, S., \& Castelli, P. (2006). Developmental differences in false-event rejection: Effects of memorability-based warnings. Memory, 14, 762-776.

Ghetti, S., Qin, J., \& Goodman, G. S. (2002). False memories in children and adults: Age, distinctiveness, and subjective experience. Developmental Psychology, 38, 705-718.

Holliday, R. E., \& Weekes, B. S. (2006). Dissociated developmental trajectories for semantic and phonological false memories. Memory, 14, 624-636.

Horton, M. S., \& Markman, E. (1980). Developmental differences in the acquisition of basic and superordinate categories. Journal of Experimental Psychology: Human Learning and Memory, 2, 322-330.

Howe, M. L. (2005). Children (but not adults) can inhibit false memories. Psychological Science, 16, 927-931.

Howe, M. L. (2006a). Developmentally invariant dissociations in children's true and false memories: Not all relatedness is created equal. Child Development, 77, 1112-1123.

Howe, M. L. (2006b). Distinctiveness effects in children's memory. In R. R. Hunt \& J. Worthen (Eds.), Distinctiveness and human memory (pp. 237-257). New York: Oxford University Press.

Howe, M. L., Cicchetti, D., Toth, S. L., \& Cerrito, B. M. (2004). True and false memories in maltreated children. Child Development, 75, 1402-1417.

Howe, M. L., Gagnon, N., \& Thouas, L. (2008). Development of false memories in bilingual children and adults. Journal of Memory and Language, 58, 669-681.

Israel, L., \& Schacter, D. L. (1997). Pictorial encoding reduces false recognition of semantic associates. Psychonomic Bulletin \& Review, 4, 577-581.

Johnson, M. K., Hashtroudi, S., \& Lindsay, D. S. (1993). Source monitoring. Psychological Bulletin, 114, 3-28.

Johnson, M. K., \& Raye, C. L. (1981). Reality monitoring. Psychological Review, 88, 67-85.

Lampinen, J. M., Leding, J. K., Reed, K. B., \& Odegard, T. N. (2006). Global gist extraction in children and adults. Memory, 14, 952-964.

Lindsay, D. S., Johnson, M. K., \& Kwon, P. (1991). Developmental changes in memory source monitoring. Journal of Experimental Child Psychology, 52, 297-318.

Lucariello, J., Kyratzis, A., \& Nelson, K. (1992). Taxonomic knowledge: What kind and when? Child Development, 63, 978-998.

Madigan, S., \& Neuse, J. (2004). False recognition and word length: A reanalysis of Roediger, Watson,
McDermott, and Gallo (2001) and some new data. Psychonomic Bulletin \& Review, 11, 567-573.

Mervis, C. B., \& Crisafi, M. A. (1982). Order of acquisition of subordinate-, basic- and superordinate-level categories. Child Development, 53, 258-266.

Metzger, R. L., Warren, A. R., Shelton, J. T., Price, J., Reed, A. W., \& Williams, D. (2008). Do children "DRM" like adults? False memory production in children. Developmental Psychology, 44, 169-181.

Nelson, D. L., McEvoy, C. L., \& Dennis, S. (2000). What is free association and what does it measure? Memory \& Cognition, 28, 887-899.

Nelson, K. (1996). Language in cognitive development: Emergence of the mediated mind. New York: Cambridge University Press.

Odegard, T. N., Holliday, R. E., Brainerd, C. J., \& Reyna, V. F. (2008). Attention to global gist processing eliminates age effects in false memories. Journal of Experimental Child Psychology, 99, 96-113.

Park, L., Shobe, K. K., \& Kihlstrom, J. F. (2005). Associative and categorical relations in the associative memory illusion. Psychological Science, 16, 792-797.

Perner, J. (2000). Memory and theory of mind. In E. Tulving \& F. I. M. Craik (Eds.), The Oxford handbook of memory (pp. 297-312). Oxford, UK: Oxford University Press.

Pesta, B. J., Murphy, M. D., \& Sanders, R. E. (2001). Are emotionally charged lures immune to false memory? Journal of Experimental Psychology: Learning, Memory Cognition, 27, 328-338.

Pierce, B. H., Gallo, D. A., Weiss, J. A., \& Schacter, D. L. (2005). The modality effect in false recognition: Evidence for test-based monitoring. Memory \& Cognition, 33, 1407-1413.

Reyna, V. F., \& Brainerd, C. J. (1995). Fuzzy-trace theory: An interitem synthesis. Learning and Individual Differences, 7, 1-75.

Roberts, K. P. (2000). An overview of theory and research on children's source monitoring. In K. P. Roberts \& M. Blades (Eds.), Children's source monitoring (pp. 11-57). Mahwah, NJ: Lawrence Erlbaum Associates Inc.

Roediger, H. L. (1996). Memory illusions. Journal of Memory and Language, 35, 76-100.

Roediger, H. L., Balota, D. H., \& Watson, J. M. (2001). Spreading activation and the arousal of false memories. In H. L. Roediger, J. S. Nairne, I. Neath, \& A. M. Surprenant (Eds.), The nature of remembering: Essays in honor of Robert G. Crowder (pp. 95115). Washington, DC: American Psychological Association.

Roediger, H. L., \& McDermott, K. B. (1995). Creating false memories: Remembering words not presented in lists. Journal of Experimental Psychology: Learning, Memory. and Cognition, 21, 803-814.

Roediger, H. L., \& McDermott, K. B. (2000). Distortions of memory. In E. Tulving \& F. I. M. Craik (Eds.), The Oxford handbook of memory. Oxford, UK: Oxford University Press.

Roediger, H. L., McDermott, K. B., Pisoni, D. B., \& Gallo, D. A. (2004). Illusory recollection of voices. Memory, 12, 586-602. 
Schacter, D. L., Israel, L., \& Racine, C. (1999). Suppressing false recognition in younger and older adults: The distinctiveness heuristic. Journal of Memory and Language, 40, 1-24.

Schacter, D. L., Kagan, J., \& Leichtman, M. D. (1995). True and false memories in children and adults: A cognitive neuroscience perspective. Psychology, Public Policy. Law, 1, 411-428.

Schacter, D. L., \& Wiseman, A. L. (2006). Reducing memory errors: The distinctiveness heuristic. In R. R. Hunt \& J. Worthen (Eds.), Distinctiveness and memory (pp. 89-107). New York: Oxford University Press.

Schneider, W., \& Bjorklund, D. (1998). Memory. In D. Kuhn \& R. Siegler (Eds.), Cognition, perception, and language [Vol. 2 of W. Damon (Series Ed.), Handbook of child psychology] (pp. 255-308). New York: Wiley.

Seamon, J. G., Luo, C. R., Schlegel, S. E., Greene, S. E., \& Goldenberg, A. B. (2000). False memory for categorised pictures and words: The category associates procedure for studying memory errors in children and adults. Journal of Memory and Language, 42, 120-146.

Skwarchuk, S., \& Anglin, J. M. (1997). Expression of superordinates in children's word definitions. Journal of Educational Psychology, 89, 298-308.

Smiley, S., \& Brown, A. L. (1979). Conceptual preferences for thematic or taxonomic relations: A nonmonotonic age trend from preschool to old age. Journal of Experimental Child Psychology, 28, 249-257.

Smith, S. M., Gerkens, D. R., Pierce, B. H., \& Choi, H. (2002). The roles of associative responses at study and semantically guided recollection at test in false memory: The Kirkpatrick and Deese hypotheses. Journal of Memory and Language, 47, 436-447.

Smith, S. M., Ward, T. B., Tindell, D. R., Sifonis, C. M., \& Wilkenfeld, M. J. (2000). Category structure and created memories. Memory \& Cognition, 28, 386-395.

Waxman, S., \& Namy, L. (1997). Challenging the notion of a thematic preference in young children. Developmental Psychology, 33, 555-567.

\section{APPENDIX}

\section{Preschoolers' lists}

\section{Categorical lists}

Animal. Lion. Elephant, giraffe, tiger, crocodile, hippopotamus, snake, zebra, dog, horse, monkey Body. Belly. Hands, legs, feet, arms, mouth, nose, eyes, head, fingers, hair

Clothing. Trousers. Sweater, shoes, coat, smock, socks, skirt, shirt, tights, panties, boots

Fruit. Apple. Pear, orange, banana, peach, pineapple, grapes, lemon, tangerine, strawberry, melon
Furniture. Table. Chair, bed, television, sofa, cupboard, lamp, clock, stool, telephone, desk Vehicle. Car. Coach, aeroplane, train, bus, motorbike, lorry, tram, boat, taxi, bicycle Associative lists

Animal. Lion. King, eats, scare, Simba, tiger, strong, jungle, mane, zoo, to roar

Body. Belly. Belly-button, heart, food, babies, breasts, pain, fat, stomach, full, hungry

Clothing. Trousers. Wear, sweater, legs, wash, shorts, shoes, to button, torn, jeans, pockets Fruit. Apple. To eat, to peel, banana, stone, pear, wash, red, peel, to chew, bite

Furniture. Table. Food, draw, games, chair, tablecloth, plates, to tidy up, clean, stool, seat

Vehicles. Car. Wheels, drive, steering-wheel, windows, doors, dad, glass, light, seats, street

\section{Preadolescents' lists}

\section{Categorical lists}

Animal. Dog. Cat, lion, giraffe, elephant, bird, dolphin, horse, monkey, cow, fish

Body. Legs. Head, arms, feet, hands, eyes, fingers, mouth, belly, ears, nose

Clothing. Trousers. Sweater, shirt, socks, t-shirt, skirt, panties, coat, shorts, sneakers, scarf

Fruit. Orange. Apple, banana, pear, pineapple, strawberry, mango, peach, kiwi, grapes, lemon

Furniture. Table. Cupboard, bed, chair, sofa, television, chest of drawers, desk, lamp, bookcase, stool

Vehicle. Car. Bus, aeroplane, train, underground, motorbike, tram, bicycle, boat, taxi, coach

\section{Associative lists}

Animal. Dog. Bitch, cat, bark, leash, collar, friend, bite, kennel, hair, doghouse

Body. Legs. To walk, to run, hair, to jump, skin, feet, long, bones, muscles, broken

Clothing. Trousers. Jeans, pockets, to dress, shorts, cords, stretched, blue, torn, zipper, tights

Fruit. Orange. Colour, juice, to eat, round, orange tree, vitamin, segment (of orange), tangerine, citrus fruit, peel

Furniture. Table. Food, wood, tablecloth, cover, chairs, dinner, lunch, meals, dish, work Vehicle. Car. Wheels, engine, steering-wheel, van, door, ride, accident, drive, street, Ferrari 